\title{
Research of Evaluation of Economic Model of Equipment Based on BP Neural Network
}

\author{
PAN Ying ${ }^{1, a}$, YANG Kuo ${ }^{2, b}$ \\ ${ }^{1}$ Equipment Academy,Beijing 101416,China \\ ${ }^{2}$ School of Automobile, Chang'an University, Xi'an Shaanxi 710064, China

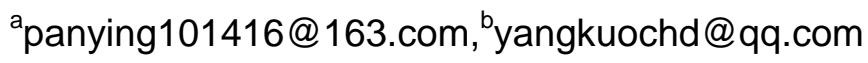

\begin{abstract}
Keywords: Equipment economy;Military equipment;BP neural network;Emergency command equipment;Comprehensive evaluation.
\end{abstract}

\begin{abstract}
The accuracy of economic evaluation on equipment demonstration projects directly affects the selection of development schemes and the amount of the investment.By analyzing the influencing factors of equipment economy,this paper established the evaluation index system and comprehensive evaluation model of equipment economy using the BP neural network.Taking a certain type of emergency command vehicle equipment as a sample,experimental results are presented to show the efficiency, accuracy and impersonality of the method.
\end{abstract}

\section{Introduction}

The development of modern equipment is not primarily constrained by technology,but subject to whether the technology advanced at the same time with high efficiency.Therefore,a comprehensive and accurate evaluation to the efficiency of equipment demonstration project is of great significance for improving scientific policy decision and saving funding[1].

Equipment economy involved in technology, economy, management, and many other factors.Economic factors that determine the size of the equipment economy is more complex,Now the economical efficiency of equipment comprehensive evaluation need to choose the reasonable evaluation index and evaluation methods[2].Equipment economic factors are very complicated, considering the cost,performance,time and risk,and these four elements exist extensive non-linear, dynamic relationship.And artificial Neural Networks is a nonlinear transform of an information system developed in recent years with a highly non-linear,intelligent feature[3].Based on the establishment of a reasonable evaluation index,through representative of the prior input "index evaluation" analysis and learning,it can make reasonable evaluation in general system.

\section{Equipment economic evaluation principle and procedure}

This paper argues that the equipment to achieve a comprehensive economic evaluation requires the demonstration phase considering cost, performance, time and risk 4 factors[4].

(1) Evaluation principle;Principle of BP neural network method for the economic evaluation of equipment is:The information of the certain type equipment economic evaluation index system as the input vector of the neural network, denoted $\mathrm{I}=\left(\mathrm{u}_{1}, \mathrm{u}_{2}, \ldots \mathrm{u}_{\mathrm{n}}\right)$; the equipment economic evaluation results as the output $\mathrm{O}$ of the neural network. With sufficient sample training the network,so that the different inputs to get different output,weight value and threshold value held by the neural network is obtained by adaptive learning correct internal representation. Trained neural network evaluation model can be used as an effective tool for the economic evaluation of equipment, in order to make different economical evaluation of alternatives.

(2) Establish evaluation index system;Establishing a scientific and reasonable system of economic evaluation index is the key to the economic evaluation of equipment.Through the analysis of factors in front of military equipment and economic evaluation of impact. Following the evaluation index system is established in this paper: the system efficiency, life cycle cost, development progress, technical risk, cost risk, schedule risk[5]. 
(3) The quantization process to determine the index of evaluation value and dimension;The technical risk of equipment economic evaluation index system in which belongs to qualitative indicators,generally by experts according to the 1-9 scale score,and the results were normalized,the performance of the system, and the cost of develop and schedule belongs to quantitative indicators,its value can be obtained by analytical calculation,cost risk and schedule risk can be obtained by expert scoring method,also can be obtained by analytical calculation[6].

(4) In 6 kinds of evaluation index, system performance belongs to the benefit index,namely,the greater the value of indicators the more beneficial for the evaluation target,the transform method: $Z_{i}^{\prime}=\frac{Z_{i}-Z_{\min }}{Z_{\max }-Z_{\min }}$.

Cost,schedule and risk index belongs to the development of the cost indexes,namely the index value smaller the more favourable to the evaluation goal,the transformation method: $z_{i}^{\prime}=\frac{Z_{\max }-Z_{i}}{Z_{\max }-Z_{\min }}$

(2) In the

formula: $Z_{i}$ is the $\mathrm{i}$ component indicators, ${ }_{i}^{\prime}$ is the normalized value of $Z_{i}, Z_{\max }, Z_{\min }$ are the minimum and maximum values.

(5) Construction of artificial neural network evaluation model; The key is to build on BP neural network to determine the number of nodes in each layer,according to the established evaluation can determine the number of neurons in the input layer for 6[7].So it can be set up the equipment and economic evaluation set $U=\{$ Excellent good general poor $\}$, said: $\{1,1\}\{1,0\}\{0,1\}\{0,0\}$. The number of neurons in the output layer is 2,This paper adopts 3 layers of BP neural network,and the number of hidden neurons is set to 5 ,so finally get the equipment economic evaluation model of neural network structure shown in Figure 1.

(6) Algorithm design; $\mathrm{U}$ as the input sample label, $\mathrm{I}_{k}^{u}(\mathrm{k}=1,2,3,4,5,6)$ as the input layer element state, $\mathrm{O}_{k}^{u}(\mathrm{i}=1,2)$ as the output layer corresponding to the unit state, $H_{j}^{u}(\mathrm{j}=1,2,3,4,5)$ is the hidden layer units corresponding state, $\mathrm{W}_{\mathrm{jk}}$ is the input layer to the hidden layer weights, $\mathrm{W}_{\mathrm{ij}}$ is the hidden layer to the output layer weights. The final output of the network for: $\left.\mathrm{O}_{i}^{u}=f_{2}\left(W_{i j} H_{j}^{u}+b_{2}\right)=f_{2}\left(W_{j k} I_{k}^{u}+b_{1}\right)+b_{2}\right)$

(3) The ideal output samples for $\left\{T_{i}^{u}\right\}$, there are 4 kinds of forms: $\{1,1\}$ is excellent; $\{1,0\}$ is good; $\{0,1\}$ is general; $\{0,0\}$ is poor;The error function and the squared error output $E(W)$ : $E(W)=\frac{1}{2} \sum_{u=1}^{m}\left(T_{i}^{u}-O_{i}^{u}\right)^{2}$

(4) In the

formula: $\mathrm{m}$ is the number of samples. By using the steepest descent method to revise the weight. 


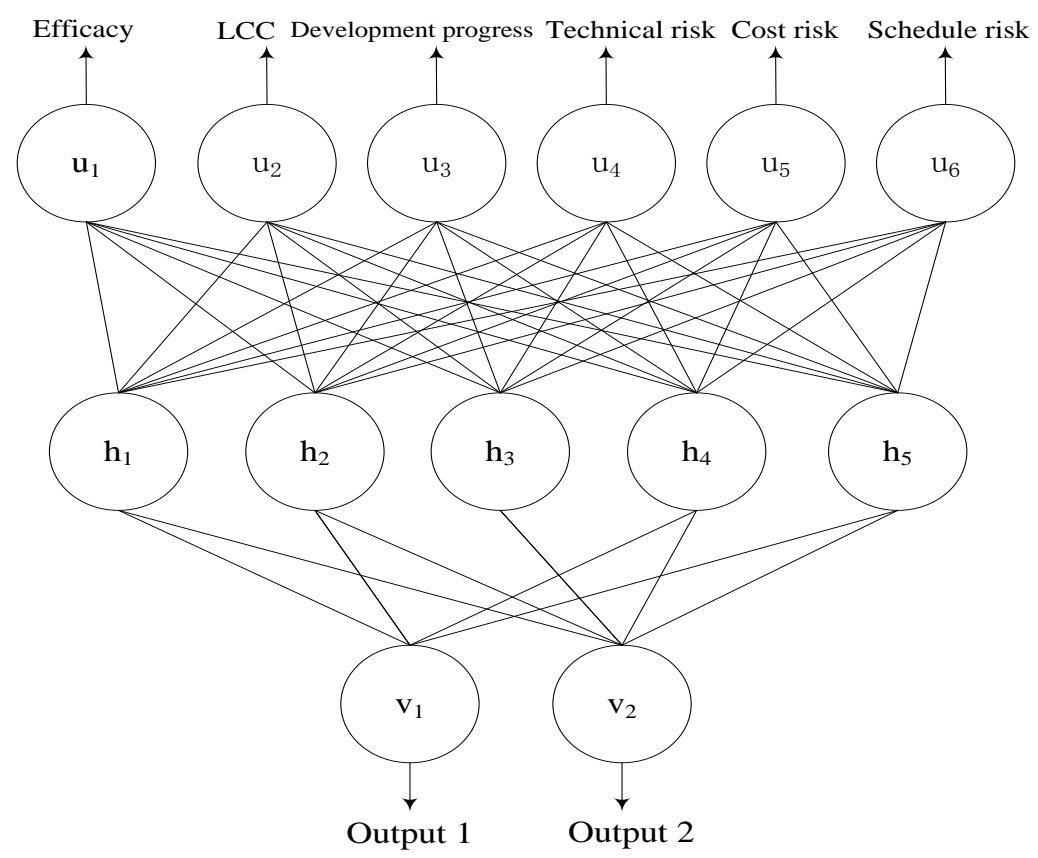

The input

layer

The hidden

layer

The output

layer

Fig.1 Neural network model of equipment economic evaluation

\section{Analysis of a certain type of emergency command vehicle equipment case}

Assuming that the amount of treated by dimensional.A type of emergency command vehicle equipment training sample information as Table 1,in the process of the establishment of evaluation model,use sigmoil function as the activation function of the hidden layer of neural network model,use purelin function as the activation function of the output layer.

Table $1 \mathrm{~A}$ type of emergency command vehicle equipment training sample data

\begin{tabular}{cccccccc}
\hline No. & Efficacy & LCC & $\begin{array}{c}\text { Development } \\
\text { progress }\end{array}$ & Technical risk & Cost risk & Schedule risk & Category \\
\hline 1 & 0.92 & 0.79 & 0.80 & 0.79 & 0.68 & 0.81 & 11 \\
2 & 0.71 & 0.77 & 0.75 & 0.67 & 0.67 & 0.72 & 10 \\
3 & 0.64 & 0.73 & 0.71 & 0.60 & 0.61 & 0.63 & 01 \\
4 & 0.53 & 0.69 & 0.81 & 0.49 & 0.77 & 0.54 & 00 \\
5 & 0.89 & 0.78 & 0.68 & 0.77 & 0.58 & 0.79 & 11 \\
6 & 0.72 & 0.76 & 0.71 & 0.69 & 0.65 & 0.71 & 10 \\
7 & 0.63 & 0.70 & 0.65 & 0.61 & 0.68 & 0.74 & 01 \\
8 & 0.56 & 0.69 & 0.83 & 0.52 & 0.88 & 0.56 & 00 \\
9 & 0.94 & 0.80 & 0.77 & 0.80 & 0.65 & 0.90 & 11 \\
10 & 0.76 & 0.71 & 0.68 & 0.70 & 0.67 & 0.66 & 10 \\
11 & 0.62 & 0.78 & 0.59 & 0.71 & 0.60 & 0.73 & 01 \\
12 & 0.48 & 0.84 & 0.59 & 0.53 & 0.83 & 0.56 & 00 \\
\hline
\end{tabular}

All learning samples input to the network was trained,until the accuracy can meet the requirements for ratio.Using the network model trained well, randomly selected a few training samples for inspection, test results are shown in Table 2, we can see that the actual output at this time has been the basic close to the ideal output.

Table 2 The trained BP neural network output results

\begin{tabular}{ccccc}
\hline Samples & The actual output & The actual output & The ideal output & The evaluation results \\
\hline Sample 1 & 1.0000 & 0.9999 & 11 & Excellent \\
Sample 4 & 0.1533 & 0.0815 & 00 & Poor \\
Sample 6 & 0.0002 & 1.0000 & 01 & Good \\
Sample 7 & 0.9271 & 0.0034 & 10 & General \\
\hline
\end{tabular}


It is assumed that there are 3 options using the model to calculate,the output of the evaluation results are shown in Table 3.With the principle of maximum membership, can be seen from table 3,evaluation result of scheme 1 as good, evaluation result of scheme 2 as excellent, evaluation result of scheme 3 as a general and should be selected scheme 2 as the implementation plan.

Table 3 Economic evaluation result of a certain type of emergency command vehicle equipment

\begin{tabular}{ccc}
\hline Schemes & Output 1 & Output 2 \\
\hline Scheme 1 & 1.0000 & 0.4839 \\
Scheme 2 & 0.9895 & 0.9490 \\
Scheme 3 & 0.0543 & 0.8754 \\
\hline
\end{tabular}

\section{Conclusion}

In this paper,a comprehensive economic evaluation of equipment were proposed,the system analyzes the main factors of economic evaluation which should be considered,on the basis of BP neural network,building a comprehensive evaluation index system of economy and military equipment and equipment economic evaluation model. When given to evaluation index information of equipment,it can be composed of model output the economic evaluation results. This method has the advantages of high efficiency,accuracy and objectivity of strong and so on.Because of the limit of training samples,the reliability of the evaluation model needs to be further improved,this will be the further study in the future,so as to continuously improve the effectiveness of equipment economic evaluation.

\section{References}

[1] Qin Z, Fu-zeng Z. Economic Evaluation of Water-conservation Equipment in Residential Building[J]. Journal of Chongqing Jianzhu University, 2007.

[2] Plaut P O. Chapter 4 - Economic and Planning Aspects of Transportation Emission[J]. Handbook of Air Pollution From Internal Combustion Engines, 1998:65-89.

[3] Yu L, Chang-bing L, Guang-yu Z. On Economy and Property of Environment Protection Equipment for Transportation Project[J]. Journal of Waterway and Harbor, 2004.

[4] Jiun F. Port Equipment Management under Market Economy.[J]. Port Operation, 1999.

[5] Wei L, Shouchun L, Tongzhou L. The Analysis and Discussion of Equipment Renewal About Technology and Economy[J]. Tactical Missile Technology, 2006.

[6] Cheng-long Y. Economy Evaluation Method of Equipment of Excavation[J]. Coal Mine Machinery, 2010.

[7] Karsak E.An overhaul-replacement model for equipment subject to technological change in inflation-prone economy[J].International Journal of Production Economics, 1998:291-301. 